MR. RUBEN PERELLÓN-ALFONSO (Orcid ID : 0000-0001-8647-3703)

Article type : Research Report

\title{
Similar effect of intermittent theta burst and sham stimulation on corticospinal \\ excitability: a 5-day repeated sessions study
}

Ruben Perellón-Alfonso ${ }^{1}$, Magdalena Kralik ${ }^{1,2}$, Indre Pileckyte $^{1}$, Matic Princic ${ }^{1}$, Jurij Bon ${ }^{1}$, Caspar Matzhold ${ }^{1,2}$, Benjamin Fischer ${ }^{1,2}$, Petra Šlahorová ${ }^{1,2}$, Zvezdan Pirtošek $^{1}$, John Rothwell ${ }^{3}$, Maja Kojovic ${ }^{1}$

${ }^{a}$ Department of Neurology, University Medical Centre Ljubljana. Zaloška 2, 1000 Ljubljana, Slovenia.

${ }^{\mathrm{b}}$ Department of Philosophy, University of Vienna. Universitätsring 1, 1010 Vienna, Austria.

${ }^{c}$ Institute of Neurology, University College London. Gower Street WC1E 6BT, London, United Kingdom.

Correspondence to:

Assoc. Prof. Maja Kojović, MD, PhD

Department of Neurology, University Medical Centre Ljubljana

Zaloška 2,1000 Ljubljana

Slovenia

Phone: +38641910 533

Email: maja.kojovic@kclj.si

This article has been accepted for publication and undergone full peer review but has not been through the copyediting, typesetting, pagination and proofreading process, which may lead to differences between this version and the Version of Record. Please cite this article as doi: 10.1111/ejn.14077

This article is protected by copyright. All rights reserved. 
Running title: Corticospinal excitability effects of 5-day iTBS

Keywords: transcranial magnetic stimulation, plasticity, motor cortex, variability

\section{Abstract}

Despite accumulating evidence of inter- and intra-individual variability in response to theta burst stimulation, it is widely believed that in therapeutic applications, repeated sessions can have a "build-up" effect that increases the response over and above that seen in a single session. However, strong evidence for this is lacking. Therefore, we examined whether daily administration of intermittent theta burst stimulation (iTBS) over the primary motor cortex induces cumulative changes in transcranial magnetic stimulation measures of cortical excitability, above the changes induced by sham stimulation. Over 5 consecutive days, 20 healthy participants received either active iTBS or sham stimulation. Each day, baseline measures of cortical excitability were assessed before and up to $30 \mathrm{~min}$ after the intervention. There was no significant difference in the rate of response between iTBS and sham stimulation on any of the 5 days. There was no iTBS specific cumulative increase of corticospinal excitability. The likelihood that an individual would remain a responder from day-to-day was low in both groups, implying high within-subject variability of both active and sham iTBS after-effects. In contrast, we found a high within-subject repeatability of resting and active motor threshold, and baseline motor evoked potential amplitude. In summary, sham stimulation has similar effect to active iTBS on corticospinal excitability, even when applied repeatedly for 5 days. Our results might be relevant to research and clinical applications of theta burst stimulation protocols. 


\section{Introduction}

Theta Burst Stimulation (TBS) protocols consist of high frequency gamma bursts, repeated at a theta frequency that can induce after-effects on the excitability of the stimulated area (Capocchi et al., 1992). In humans, when TBS is applied intermittently (iTBS) over the primary motor cortex (M1), it may facilitate corticospinal excitability (long term potentiation-like effect), conversely, when applied continuously (cTBS), it may decrease corticospinal excitability (long term depression-like effects) (Huang et al., 2005). TBS protocols have gained attention during the last decade, because they are quick to apply, require sub-threshold stimulation intensities, and are therefore more practical and potentially safer to administer than classical high and low frequency repetitive transcranial magnetic stimulation (rTMS) protocols (Huang et al., 2005; Daskalakis, 2014; Suppa et al., 2016). Recent evidence suggests that TBS protocols have a variable effect on cortical excitability among different subjects, with a high proportion of subjects either not responding or responding in the opposite direction from what is expected (Hamada et al., 2013; Lopez-Alonso et al., 2014; Vernet et al., 2014; Vallence et al., 2015; Hordacre et al., 2017). This calls into question both the efficacy of theta burst stimulation as an experimental method for probing brain plasticity and its usefulness as a therapeutic tool. The within-subject variability of the rTMS after-effect has been less investigated, however, it is considered to be lower than the between-subject variability (Sommer et al., 2002). Numerous studies have tried to define potential sources of variability, including gender differences (Vernet et al., 2014), genetic factors (Hoppenrath \& Funke, 2013), early life events (Pitcher et al., 2012), target muscle pre-activation (Gentner et al., 2008; lezzi et al., 2008) and time of the day when experiments are performed (Sale et al., 2008). Other suggested factors are: history of synaptic plasticity (Murakami et al., 2012), between-subject 
differences in intracortical neurons that are activated by stimualtion (Hamada et al., 2013) or differences in functional connectivity (Nettekoven et al., 2014; Nettekoven et al., 2015). However, none of these factors seem sufficient to explain all the variance observed. Little is known about the contribution of the placebo effect to the overall response to rTMS protocols (Duecker \& Sack, 2015) and, in particular, about its contribution to the variability of the rTMS response.

The issue of the efficacy of rTMS as a plasticity-inducing tool is of clinical importance. It is widely believed that a clinically significant response to therapeutic applications of rTMS occurs if sessions of rTMS are repeated over a period of several days (Lefaucheur et al., 2014). This "build-up" effect presumably results from cumulative brain changes that potentiate the effect of single stimulations. The evidence supporting cumulative effects has been scarce, both in animal studies (Valero-Cabre et al., 2008) and in patient populations (Khedr et al., 2006; Avenanti et al., 2012; Kimberley et al., 2013; Munneke et al., 2013; Kim et al., 2015), and the mechanism through which this might be occurring remains uninvestigated.

In the present study, we tested whether daily administration of iTBS over the M1 produces cumulative changes in transcranial magnetic stimulation (TMS) measures of cortical excitability, over and above the changes induced by sham stimulation. We hypothesized that cumulative changes would cause progressive increase of iTBS after-effects in individual volunteers and/or increase the number of subjects who respond to iTBS.

Consecutive days of stimulation were chosen to imitate the design usually applied in clinical studies (Lefaucheur et al., 2014). In order to mimic the anticipatory expectations of patients undergoing such protocols in the clinical setting, we did not inform participants that there was a sham treatment group. In fact, irrespective of the protocol received, participants were 
told that gradual change in excitability over the week is to be expected. Our hypothesis was that if iTBS has a specific cumulative effect, then the response over 5 days to active stimulation should be greater than the response to sham stimulation. Conversely, if there is no specific effect, then, any changes over time (either increases or decreases) should be equally evident in both groups. In order to account for the possibility that effects of repeated iTBS stimulation occur by modifying levels of intracortical excitability (Ziemann \& Siebner, 2008; Siebner, 2010), measures of intracortical inhibition and facilitation were also recorded.

\section{Materials and Methods}

\section{Participants}

20 healthy volunteers ( 7 males, 13 females), aged between 18 and 31 years $(M=23.3, S D=$ 3.5) participated in the study. One participant was left handed, as determined by the Edinburgh Handedness Inventory (Oldfield, 1971). All procedures were performed in accordance with the Declaration of Helsinki and were approved by the National Medical Ethics Committee of Slovenia. All subjects gave written informed consent.

\section{Electromyography recording}

Participants were seated in a comfortable chair not facing the computer screen.

Electromyographic activity (EMG) was recorded from the dominant first dorsal interosseous (FDI) muscle using disposable bipolar silver-silver chloride electrodes. EMG was band-pass filtered $(20-2000 \mathrm{~Hz})$ and amplified (gain 1000) using a D360 amplifier (Digitimer Ltd, Welwyn Garden City, UK). The amplified EMG was sampled at $5 \mathrm{kHz}$ using a Micro1401-3

This article is protected by copyright. All rights reserved. 
data acquisition unit (Cambridge Electronic Design Limited, Cambridge, UK) coupled with Signal 5.1 software (Cambridge Electronic Design Limited, Cambridge, UK)

\section{Transcranial Magnetic Stimulation}

Transcranial magnetic stimulation was applied over the FDI muscle representation in the M1, contralateral to the target muscle. The FDI 'hotspot' was defined as the position at which single pulse TMS produced the largest motor evoked potentials (MEPs) in the contralateral FDI muscle. Single and paired pulse TMS was administered using Magstim 200 stimulators with a $70 \mathrm{~mm}$ figure-of-eight coil, while TBS was administered using a Magstim Rapid2 with an air-cooled $70 \mathrm{~mm}$ figure-of-eight coil (Magstim Company, Whitland, UK). The coil was held tangentially to the scalp, with the handle pointing posteriorly at a 45-degree angle with the midline, which is optimal for evoking electrical currents in a posterioranterior direction within the motor cortex.

\section{Corticospinal excitability}

Active motor threshold (AMT) was defined as the minimum stimulus intensity evoking a discernible MEP ( $>200 \mu \mathrm{V}$ amplitude) in at least half of 6 trials during mild contraction (approximately $10-20 \%$ of maximum voluntary contraction) of the FDI muscle. Resting motor threshold (RMT) was defined as the minimum stimulus intensity evoking a discernible MEP ( $>50 \mu \mathrm{V}$ amplitude) in at least half of 6 trials. AMT was measured using the Magstim Rapid2 stimulator, while RMT was measured using the Magstim 200 stimulator.

Baseline MEP amplitude was assessed by recording 20 MEPs from the FDI muscle at an intensity of $120 \%$ of RMT (Rossini et al., 2015). After either active or sham iTBS, 7 blocks of 
20 MEPs at $120 \%$ of baseline RMT were recorded, first immediately after iTBS and then every five minutes up to 30 minutes after iTBS.

\section{Intracortical excitability}

Short-latency intracortical inhibition (SICI) and intracortical facilitation (ICF) were recorded at rest using a standard paired-pulse paradigm (Kujirai et al., 1993). The intensity of the conditioning stimulus was set at $80 \%$ of $A M T$, whereas the intensity of the test stimulus was set at $120 \%$ of RMT. SICl and ICF were assessed by delivering 10-paired pulses at 2, 3, 4 and 15 ms inter-stimulus intervals (ISIs) and 10 test single pulses, in a randomized fashion.

\section{Active and sham iTBS techniques}

Active iTBS involved $2 \mathrm{~s}$ trains of TBS ( 3 pulses at $50 \mathrm{~Hz}$ repeated at $200 \mathrm{~ms}$ intervals) that occurred every $10 \mathrm{~s}$ for a total of $190 \mathrm{~s}$ (600 pulses). Stimulation intensity of the theta burst interventions was set to $80 \%$ of AMT in each session (Huang et al., 2005). Sham iTBS was delivered with the same parameters as in the active group but with the coil tilted so that only the side of the right edge of the coil remains in contact with the scalp at the FDI hot spot (Lisanby et al., 2001). This way, the subject can still hear the clicking sound the coil emits and feel the pressure of the coil, but the magnetic field strength is weakened enough not to induce any current in brain tissue. During both types of interventions, an experimenter ensured that participants kept their arms relaxed and looked passively forwards. 


\section{Experimental design}

Participants were told that cortical excitability would be measured first and, then, a plasticity protocol would be applied to try and temporarily increase cortical excitability. They were told that, in order to study possible cumulative effects, the same procedure would be repeated for five consecutive days. Participants were not aware that half of them would receive sham stimulation. All participants were naïve to TMS as they had not participated in any TMS study previously. Participants were randomly assigned to either active or sham iTBS. For each individual participant, five consecutive experimental sessions were conducted at the same time of day, in order to control for any diurnal effects on corticospinal plasticity. All participants were stimulated between 9 am and 2 pm and both groups where matched for the number of subjects that did the experiment before or after $12 \mathrm{pm}$. In each session, we measured RMT, AMT and MEPs. Then, active or sham iTBS was delivered and MEPs re-measured up to $30 \mathrm{~min}$, every 5 minutes. SICI and ICF were recorded before active and sham iTBS, only on days 1 and 5 (Figure 1 ).

\section{Definition of responders}

We defined "responders" and "non-responders", in both active and sham groups, according to the change in average MEP amplitude after stimulation. To exclude random fluctuations around the baseline level, we used the cut-off values of $10 \%$ and $20 \%$. Subjects were classified as "responders" if, after stimulation, they showed increase in average MEP amplitude $>10 \%$ (or $>20 \%$ ) compared to baseline average MEP amplitude (Hinder et al., 2014; Nettekoven et al., 2015), otherwise they were considered "non-responders". We also defined as "opposite responders" those subjects who showed a decrease $>10 \%$ (or $>20 \%$ ) from baseline (Table 1). 


\section{Data analysis}

Cortical excitability and paired pulse protocols data was preprocessed using our own custom made $\mathrm{C}++$ analysis software. Further data manipulations were performed using custom made Matlab (MathWorks, Inc. Chicago) scripts. Statistical analysis and resulting figures and tables were done in R using RStudio (RStudio, Inc. Boston, MA).

We first ensured that there was no muscle activity prior to each TMS pulse. Trials in which the root mean square amplitude of the 100 ms prior to the TMS pulse was larger than 50 $\mu \mathrm{V}$, were excluded from further analysis. Peak to peak amplitudes were measured for each trial, averaged for each block and paired pulse ISI (for SICI and ICF) and expressed as a ratio of the baseline or test stimuli mean, respectively. Ratio normalization was carried out according to the formula: $\frac{\overline{M E P ~ B L O C K ~ O R I S I}-\overline{\text { BASELINE OR TEST STIMULI }}}{\overline{B A S E L I N E ~ O R T E S T ~ S T I M U L I}}$ (Sinclair \& Hammond 2009; Hinder et al., 2014). Shapiro-Wilk test ( $W=0.834, p<.001)$, assessment of kurtosis (10.9) and skewness (SE=2.37) indicated that normalized average MEP amplitudes were not normally distributed and were skewed. Therefore, natural logarithm transformation was applied to all normalized average data (Sinclair \& Hammond, 2009). Means and standard deviations reported in this paper are back-transformed.

\section{Statistical analysis}

A mixed design ANOVA was used to investigate the differences between the active iTBS and sham groups for each of the measures of interest (RMT, AMT, post-iTBS MEPs and SICI/ICF across the 5 days). When a significant main effect was found, pairwise analysis with Bonferroni correction was used. Contingency tables and Fisher's exact test (FET) were used to determine the independence of the response rate from day 1 and day 5, as well as the independence of the response rate between groups (iTBS vs. sham). The analysis was 
performed separately for each cutoff (10\% and $20 \%$ ). The reliability of RMT, AMT and baseline MEPs, across the 5 days, was assessed with Intraclass Correlation Coefficient estimates based on mean rating $(k=5)$, absolute-agreement, two-way random-effects model, ICC $(2, k)$. We did not use ICC to assess the repeatability of active and sham iTBS induced changes in corticospinal excitability (after-effect) or for the repeatability of SICI and ICF, because these measures are expressed as a ratio of the baseline and test stimulus, respectively. Normalization of the data reduces the total variance of the data, and thus reduces the ICC estimates of variability, rendering ICC a non-suitable measure for repeatability of normalized data (Koo \& Li, 2016). In order to assess within-subject variability in the response to active and sham iTBS, we used Fleiss' kappa (Fleiss, 1971), for each group and cutoff separately. This test assesses the reliability of the binary stimulation effect (responder vs. non-responder) in the same participant across the 5 days.

\section{Results}

Effects of active and sham iTBS on corticospinal excitability Mixed design ANOVA on RMT with one within-subject factor (day, 5 levels) and one between-subject factor (group, 2 levels), revealed a significant main effect of day $\left(F(4,72)=5.30, p=.001, \eta^{2}=.013\right)$, but not of group. Post-hoc analysis revealed that RMT was significantly larger in day $1(M=44.5, S D=7.37, p=.014)$ than in day $2(M=42.9, S D=7.39)$. There was no significant interaction between group and day. Similarly, mixed design ANOVA on AMT revealed a significant main effect of day $\left(F(4,72)=2.84, p=.030, \eta^{2}=.015\right)$, but not of group. There was no significant interaction between the two factors. Post hoc analysis with Bonferroni correction did not reveal any significant differences between days due to the relatively small effect size. 
To assess possible differences in baseline MEP amplitude between groups, we used mixed design ANOVA with a within-subject factor (day, 5 levels) and a between subject factor (group, 2 levels), on log transformed average baseline MEP amplitude. This analysis revealed a significant main effect of day, $\left(F(4,72)=2.30, p=.043, \eta^{2}=.6\right)$, but not of group, or their interaction. However, post hoc analysis did not reveal any significant differences between the days due to the relatively small effect. Moreover, the mean amplitude of the baseline was $0.891 \mathrm{mV}(S D=1.67)$ and $0.890 \mathrm{mV}(S D=1.87)$ in the active and sham iTBS groups, respectively. These results indicate that baseline MEP amplitude did not differ between groups on any of the days of stimulation.

To assess the differences between the effects of the active and sham iTBS groups on MEP amplitude, we performed mixed design ANOVA on log normalized average post-iTBS MEP amplitude with two within-subject factors (day, 5 levels, and block, 7 levels) and a betweensubject factor (group, 2 levels). The analysis revealed a significant main effect of day $(F(4$, $\left.72)=3.64, p=.009, \eta^{2}=.079\right)$. Post hoc analysis with Bonferroni correction revealed this was due to log transformed normalized average MEP amplitude on day 5 being significantly $\operatorname{larger}(M=0.351, S D=0.56)$ than on days 1 to $4(M=0.129, S D=0.503, p=.004 ; M=0.004$, $S D=0.635, p<.001 ; M=0.135, S D=0.569, p=.003 ; M=-0.071, S D=0.563, p<.001)$. Additionally, log transformed normalized average MEP amplitude on day 4 was significantly smaller than in day $1(p=.004)$ and day $3(p=.001)$. The main effect of group and block, as well as their interaction, was not significant, indicating that the effect of stimulation on corticospinal excitability was the same in both sham and active groups (Figure 2). Given the variability in mean effect across days, we conclude that there was no evidence for a general upward trend in the data over the consecutive days.

This article is protected by copyright. All rights reserved. 


\section{$\mathrm{SICl}$ and ICF}

Mixed design ANOVA on SICl with two within-subject factors (day, 2 levels; ISI, 3 levels) and a between-subject factor (group, 2 levels) revealed a statistically significant main effect of ISI $(F(3,54)=10.86, p=.001)$. This was due to less $S I C l$ at $4 \mathrm{~ms} I S I(M=-0.189, S D=0.718)$ than at 2 $\mathrm{ms}$ ISI $(M=-0.390, S D=0.732, p=.002)$ and $3 \mathrm{~ms} I S I(M=-0.389, S D=0.940, p=.001)$. There was no significant main effect of day and group, as well as no significant interactions (Figure 3). Mixed design ANOVA on ICF, with a within-subject factor (day, 2 levels) and a betweensubject factor (group, 2 levels), revealed no significant main effect or interaction.

These results indicate that the two groups did not differ in baseline $\mathrm{SICl}$ and ICF on the first day of stimulation, and that a 4-day course of either active or sham iTBS did not affect intracortical inhibition or facilitation.

Reliability of the baseline TMS measures across days

Across the 5 sessions, ICC indicated high and significant reliability of baseline average MEP amplitude (ICC=.776, with $95 \%$ confidence interval from .576 to $.899, F(19,74.4)=4.71$, $p<.001)$ and very high and significant reliability of both RMT (ICC $=.986$ with $95 \%$ confidence interval from .986 to $.994, F(19,50.6)=85.9, p<.001)$ and AMT $(I C C=.97$, with $95 \%$ confidence interval from .942 to $.986, F(19,71.3)=35.7, p<.001)$. RMT and AMT values for each day are given in Table 2.

\section{Variability of the response to active and sham iTBS}

To assess differences in the overall number of responders between groups, we first expressed the raw average post-iTBS MEP amplitude in all blocks as a percentage of baseline, then we averaged together all post-iTBS time blocks for each day in every 
participant and coded them as either responders or non-responders for each cutoff separately. Contingency table (active vs. sham) and Fisher's exact test revealed no significant difference in the rate of response between the active and sham iTBS groups, neither for $10 \%(p=.106, F E T)$ nor $20 \%(p=.106, F E T)$ cutoff (Table 1$)$.

The relationship between the rate of response on days 1 and 5 was assessed for each group and cutoff separately. Fisher's exact test revealed no significant change in response rate from day 1 to day 5 in either group, for any of the cutoff values (iTBS, 10\% cutoff: $p=1,20 \%$ cutoff: $p=1$; sham, $10 \%$ cutoff: $p=.58,20 \%$ cutoff: $p=.35$ )

The likelihood that an individual would remain a responder from day-to-day was low in both active and sham iTBS groups, for either $10 \%(\mathrm{k}=.159, p=.113$ and $\mathrm{k}=-.07, p=.487$, for active and sham groups respectively), or for $20 \%(\mathrm{k}=.02, p=0.845$ and $\mathrm{k}=.039, p=.701$, for active and sham groups respectively) cutoff values.

\section{Discussion}

We report four main findings of the study. (i) The effect of active iTBS was not different from the effect of sham stimulation on any of the 5 days. (ii) Repeated sessions of iTBS did not produce a cumulative effect (over and above placebo) on corticospinal excitability, neither did they change the number of "responders". (iii) There were some day-to-day changes in the response to both active and sham iTBS, however, these were the same in both groups. (iv) AMT, RMT and baseline MEPs were highly repeatable measures across the different sessions.

This article is protected by copyright. All rights reserved. 
The effect of active and sham iTBS on corticospinal excitability

There are two novel findings in the present data. First, even over a 5-day "treatment" protocol, active iTBS was not superior to sham stimulation at inducing long-lasting facilitation of corticospinal excitability. Second, there was no evidence, in either group, of a steady increase in the response to iTBS. These conclusions were the same whether we analyzed the whole group data or separated the participants into "responders" and "nonresponders".

As noted in the Introduction, any specific cumulative effect of iTBS should have been evident as a gradual separation between the iTBS and sham groups, in their response over the 5 days. The fact that this was not observed, suggests that either there is no specific effect of iTBS or that it was too small for us to observe with our experimental design. Despite the lack of a group difference, there was an overall effect of time in that the response to both active and sham iTBS changed over the 5 days, being smaller on day 4 and larger on day 5, compared to day 1 . It is possible that some of these non-specific effects of time were the result of expectations. For example, the awareness that the day 5 was the last day of stimulation could have resulted in an increase of MEP amplitude, considering that subjects were told in advance that the procedure will be repeated for 5 consecutive days in order to study possible cumulative effects. However, this is only speculation, as we did not obtain measures of expectation, to test its relationships with TMS effects.

\section{Between and within-subject variability in response to iTBS}

Although initial studies reported a clear facilitatory after-effect of single session iTBS on corticospinal excitability (Huang et al., 2005, Huang et al. 2007), recent studies using larger samples of healthy subjects have shown a lack of group effect to both iTBS and cTBS, as well 
as to other experimental plasticity protocols (Hamada et al., 2013; Lopez-Alonso et al., 2014). This is apparently due to large between-subject variability in response to noninvasive brain stimulation (NIBS) protocols. The source of variability in response to NIBS remains largely unresolved, but differences between subjects in intrinsic mechanisms of synaptic plasticity (Ridding \& Ziemann, 2010) might be involved. Alternatively, it has been suggested that variability may be the result of preferential stimulation of certain populations of cortical neurons or differences in pre-stimulation level of excitability of the neurons (Day et al., 1989; Rothwell, 1997).

One way to quantify the inter-individual variability of iTBS effects is to measure the proportion of "responders" and non-responders" to the protocol. In the study by Hamada et al. (2013), the responses of 52 individuals to both iTBS and cTBS were highly variable, with overall no significant changes in corticospinal excitability after TBS at the group level. When the response to iTBS was defined operationally, according to whether the grand average effect was above or below 1 , authors found almost equal numbers ( $52 \%$ vs. $48 \%$ ) of participants who responded in the expected (facilitatory) as in the opposite (inhibitory) direction. In the present paper, we used a more rigorous definition of "responders" which was introduced by Nettekoven et al. (2015). "Responders" are defined as participants who have a post-iTBS increase in MEP amplitude $10 \%$ or $20 \%$ above baseline. When we applied these criteria to both groups, we found no significant difference in the number of responders to active and sham iTBS from day 1 to day 5 (Table 1). At the lower cutoff of $10 \%$, on the first day of stimulation, there were $60 \%$ of "responders "in the active group and $70 \%$ of responders in the sham group. Increasing the cutoff value to $20 \%$ revealed similar findings, with $40 \%$ of responders in the active and $50 \%$ in the sham group. A similar situation occurred on each of the 5 days. Even though the number of participants in our 
groups was not high, the observed ratio of responders is very similar to the findings of Hamada et al. (2013), including the proportion of individuals responding in the direction opposite to what is expected (Table 1). The novel finding of our study is that there was the same frequency of responders in the active and sham iTBS groups.

Day-to-day (within-subject) variability of the response to active and sham iTBS For the purposes of the present experiment, the most important measure is the day-to-day intra-individual variation in response to iTBS. Few previous studies have examined whether the effect of TBS on the motor cortex is repeatable in the same subjects on different occasions (Hinder et al., 2014; Vallence et al., 2015; Fried et al., 2017). Even though these studies found that the effect of TBS may be similar on different days at a group level, within individual repeatability was low in the latest studies (Vallence et al., 2015; Fried et al., 2017), as estimated by low ICC value. However, ICC can underestimate the repeatability of data which is expressed as a ratio, that is, normalization of the data reduces the total variance of the data, which in turn, reduces the ICC estimates of variability (Koo \& Li, 2016). For this reason, we tested the repeatability of active and sham iTBS induced changes on corticospinal excitability using Fleiss' kappa instead. This test allowed us to assess whether the same subject behaved in a similar way over the 5 days. As indicated by a low Fleiss' kappa and illustrated in Figure 4, we found low reproducibility. In other words, a participant could be a "responder" one day and a "non-responder" another day. Reconciling our data with findings from previous studies, we conclude that, at an individual level, the response to TBS (and probably to NIBS in general) is not a predetermined characteristic of the motor cortex (Day et al., 1989; Rothwell, 1997).

This article is protected by copyright. All rights reserved. 
The effect of active and sham iTBS on intracortical excitability

We found no effect on intracortical facilitation or inhibition, after 4 days of either active iTBS or sham stimulation, as measured by changes in SICl and ICF between the $1^{\text {st }}$ and $5^{\text {th }}$ day. A recent meta-analysis (Chung et al. 2016) revealed no changes in SICI or ICF after a single session of iTBS. Our results further suggest that there is no cumulative effect of repeated sessions of iTBS on intracortical inhibition and facilitation.

\section{Reliability of other measures of interest}

In our study, RMT and AMT were the most reliable measures of cortical excitability as defined by ICC. This strengthens the findings of previous studies (Ngomo et al., 2012; Liu \& Au-Yeung, 2014), which measured the same parameters on two occasions only. We also found that average baseline MEPs, measured at $120 \%$ of RMT, are relatively stable in our participants across days.

\section{Study limitations}

The main limitation of our study is the relatively small number of participants in each group, However, it is noteworthy that the measurements were performed 5 times in each participant, totaling to 100 separate experiments/observations. Moreover, since the two groups did not differ in any of the measures of interest, the analysis of reliability could be performed on pooled data, therefore increasing the statistical power of the analysis.

This article is protected by copyright. All rights reserved. 


\section{Possible relevance of our findings}

The fact that there were no differences between active and sham iTBS in our study, coupled with the fact that there was a similar proportion of responders in both groups, suggests that placebo effect might play an important role in the response to rTMS. The placebo effect is closely related to expectations. Laboratory and clinical study have shown that, when people ingest a pharmacologically inert substance, but believe that it is an active substance, they experience measurable physiologic effects expected from that active substance (Benedetti et al., 2010). Therefore, a placebo response to rTMS is not surprising, particularly when considering the robustness and impressiveness of the TMS and EMG equipment. It is worth noticing that a recent meta-analysis of studies in depression showed that although the response rate (defined $\geq 50 \%$ reduction in post-treatment depression scores) was significantly higher in iTBS treated patients, remission rates were not significantly different between patients treated with active and sham stimulation (Berlim et al., 2017).

\section{Conclusions}

We performed the first sham-controlled study investigating the effects of iTBS on corticospinal excitability across 5 consecutive days. The results suggest that iTBS is not better suited than sham to produce long-lasting facilitation of corticospinal excitability. We also investigated possible cumulative effects of repeated (5 days) stimulation and, while there were small changes between days, all effects were present in equal magnitude in both groups. RMT, AMT and baseline MEPs were highly repeatable across 5 consecutive days. On the other hand, iTBS after-effects had low repeatability, suggesting high within-subject variability. The results of this study are relevant for both research and clinical applications of 
TBS plasticity protocols and further stress the need to conduct large-scale sham-controlled studies.

\section{Acknowledgements}

This study was partially funded by the Slovenian Research Agency Grant J3-7320.

\section{Conflict of interest}

None of the authors report any potential conflict of interest.

\section{Author contributions}

RPA, JB, MK, and ZP contributed to the conception and design of this study. RPA, MK, IP, $\mathrm{MP}, \mathrm{CM}, \mathrm{BF}$, and PS collected the data and RPA and IP analyzed the data. RPA, MK, and JR drafted the manuscript, and $M K, I P, M P, J B, C M, B F, P S$, and $Z P$ were involved in revising the article. All authors have approved the final version of the manuscript to be published.

\section{Data accessibility}

Data can be requested from the corresponding author.

\section{Abbreviations}

AMT, active motor threshold; cTBS, continuous theta burst stimulation; EMG, electromyography; FDI, first dorsal interosseous; FET, Fisher's exact test; ICC, intraclass correlation coefficient; ICF, intracortical inhibition; ISI, inter-stimulus interval; iTBS, intermittent theta burst stimulation; M1, primary motor cortex; MEP, motor evoked potential; NIBS, non-invasive brain stimulation; RMT, resting motor threshold; rTMS, 
repetitive transcranial magnetic stimulation; $\mathrm{SICl}$, short interval intracortical inhibition; TBS,

theta burst stimulation; TMS, transcranial magnetic stimulation

\section{References}

Avenanti, A., Coccia, M., Ladavas, E., Provinciali, L. \& Ceravolo, M.G. (2012) Low-frequency rTMS promotes use-dependent motor plasticity in chronic stroke: a randomized trial. Neurology, 78, 256-264.

Benedetti, F., Carlino, E. \& Pollo, A. (2010) How Placebos Change the Patient's Brain. Neuropsychopharmacology, 36, 339.

Bergmann, T.O., Molle, M., Schmidt, M.A., Lindner, C., Marshall, L., Born, J. \& Siebner, H.R. (2012) EEG-guided transcranial magnetic stimulation reveals rapid shifts in motor cortical excitability during the human sleep slow oscillation. J Neurosci, 32, 243-253.

Berlim, M.T., McGirr, A., Rodrigues Dos Santos, N., Tremblay, S. \& Martins, R. (2017) Efficacy of theta burst stimulation (TBS) for major depression: An exploratory meta-analysis of randomized and sham-controlled trials. J Psychiatr Res, 90, 102-109.

Capocchi, G., Zampolini, M. \& Larson, J. (1992) Theta burst stimulation is optimal for induction of LTP at both apical and basal dendritic synapses on hippocampal CA1 neurons. Brain research, 591, 332-336.

Chung, S.W., Hill, A.T., Rogasch, N.C., Hoy, K.E. \& Fitzgerald, P.B. (2016) Use of theta-burst stimulation in changing excitability of motor cortex: a systematic review and metaanalysis. Neuroscience \& Biobehavioral Reviews, 63, 43-64.

Daskalakis, Z.J. (2014) Theta-burst transcranial magnetic stimulation in depression: when less may be more. Brain, 137, 1860-1862.

Day, B.L., Dressler, D., Maertens de Noordhout, A., Marsden, C.D., Nakashima, K., Rothwell, J.C. \& Thompson, P.D. (1989) Electric and magnetic stimulation of human motor cortex: surface EMG and single motor unit responses. The Journal of Physiology, 412, $449-473$.

Duecker, F. \& Sack, A.T. (2015) Rethinking the role of sham TMS. Front Psychol, 6, 210.

Ferreri, F., Vecchio, F., Ponzo, D., Pasqualetti, P. \& Rossini, P.M. (2014) Time-varying coupling of EEG oscillations predicts excitability fluctuations in the primary motor cortex as reflected by motor evoked potentials amplitude: an EEG-TMS study. Human brain mapping, 35, 1969-1980.

Fleiss, J.L. (1971) Measuring nominal scale agreement among many rater. Psychological Bulletin, 76, 378-382.

This article is protected by copyright. All rights reserved. 
Fried, P.J., Jannati, A., Davila-Perez, P. \& Pascual-Leone, A. (2017) Reproducibility of SinglePulse, Paired-Pulse, and Intermittent Theta-Burst TMS Measures in Healthy Aging, Type-2 Diabetes, and Alzheimer's Disease. Front Aging Neurosci, 9, 263.

Gentner, R., Wankerl, K., Reinsberger, C., Zeller, D. \& Classen, J. (2008) Depression of human corticospinal excitability induced by magnetic theta-burst stimulation: evidence of rapid polarity-reversing metaplasticity. Cereb Cortex, 18, 2046-2053.

Hamada, M., Murase, N., Hasan, A., Balaratnam, M. \& Rothwell, J.C. (2013) The role of interneuron networks in driving human motor cortical plasticity. Cereb Cortex, 23, 1593-1605.

Hinder, M.R., Goss, E.L., Fujiyama, H., Canty, A.J., Garry, M.I., Rodger, J. \& Summers, J.J. (2014) Inter- and Intra-individual variability following intermittent theta burst stimulation: implications for rehabilitation and recovery. Brain Stimul, 7, 365-371.

Hoppenrath, K. \& Funke, K. (2013) Time-course of changes in neuronal activity markers following iTBS-TMS of the rat neocortex. Neurosci Lett, 536, 19-23.

Hordacre, B., Goldsworthy, M.R., Vallence, A.M., Darvishi, S., Moezzi, B., Hamada, M., Rothwell, J.C. \& Ridding, M.C. (2017) Variability in neural excitability and plasticity induction in the human cortex: A brain stimulation study. Brain Stimul, 10, 588-595.

Huang, Y.Z., Chen, R.S., Rothwell, J.C. \& Wen, H.Y. (2007) The after-effect of human theta burst stimulation is NMDA receptor dependent. Clin Neurophysiol, 118, 1028-1032.

Huang, Y.Z., Edwards, M.J., Rounis, E., Bhatia, K.P. \& Rothwell, J.C. (2005) Theta burst stimulation of the human motor cortex. Neuron, 45, 201-206.

lezzi, E., Conte, A., Suppa, A., Agostino, R., Dinapoli, L., Scontrini, A. \& Berardelli, A. (2008) Phasic voluntary movements reverse the aftereffects of subsequent theta-burst stimulation in humans. J Neurophysiol, 100, 2070-2076.

Khedr, E.M., Rothwell, J.C., Shawky, O.A., Ahmed, M.A. \& Hamdy, A. (2006) Effect of daily repetitive transcranial magnetic stimulation on motor performance in Parkinson's disease. Movement Disorders, 21, 2201-2205.

Kim, M.S., Chang, W.H., Cho, J.W., Youn, J., Kim, Y.K., Kim, S.W. \& Kim, Y.H. (2015) Efficacy of cumulative high-frequency rTMS on freezing of gait in Parkinson's disease. Restor Neurol Neurosci, 33, 521-530.

Kimberley, T.J., Borich, M.R., Arora, S. \& Siebner, H.R. (2013) Multiple sessions of lowfrequency repetitive transcranial magnetic stimulation in focal hand dystonia: clinical and physiological effects. Restor Neurol Neurosci, 31, 533-542.

Koo, T.K. \& Li, M.Y. (2016) A Guideline of Selecting and Reporting Intraclass Correlation Coefficients for Reliability Research. Journal of Chiropractic Medicine, 15, 155-163.

Kujirai, T., Caramia, M.D., Rothwell, J.C., Day, B.L., Thompson, P.D., Ferbert, A., Wroe, S., Asselman, P. \& Marsden, C.D. (1993) Corticocortical inhibition in human motor cortex. J Physiol, 471, 501-519.

This article is protected by copyright. All rights reserved. 
Lefaucheur, J.P., Andre-Obadia, N., Antal, A., Ayache, S.S., Baeken, C., Benninger, D.H., Cantello, R.M., Cincotta, M., de Carvalho, M., De Ridder, D., Devanne, H., Di Lazzaro, V., Filipovic, S.R., Hummel, F.C., Jaaskelainen, S.K., Kimiskidis, V.K., Koch, G., Langguth, B., Nyffeler, T., Oliviero, A., Padberg, F., Poulet, E., Rossi, S., Rossini, P.M., Rothwell, J.C., Schonfeldt-Lecuona, C., Siebner, H.R., Slotema, C.W., Stagg, C.J., VallsSole, J., Ziemann, U., Paulus, W. \& Garcia-Larrea, L. (2014) Evidence-based guidelines on the therapeutic use of repetitive transcranial magnetic stimulation (rTMS). Clin Neurophysiol, 125, 2150-2206.

Lisanby, S.H., Gutman, D., Luber, B., Schroeder, C. \& Sackeim, H.A. (2001) Sham TMS: intracerebral measurement of the induced electrical field and the induction of motor-evoked potentials. Biological psychiatry, 49, 460-463.

Liu, H. \& Au-Yeung, S.S. (2014) Reliability of transcranial magnetic stimulation induced corticomotor excitability measurements for a hand muscle in healthy and chronic stroke subjects. J Neurol Sci, 341, 105-109.

Lopez-Alonso, V., Cheeran, B., Rio-Rodriguez, D. \& Fernandez-Del-Olmo, M. (2014) Interindividual variability in response to non-invasive brain stimulation paradigms. Brain Stimul, 7, 372-380.

Moliadze, V., Fritzsche, G. \& Antal, A. (2014) Comparing the efficacy of excitatory transcranial stimulation methods measuring motor evoked potentials. Neural Plast, 2014, 837141.

Munneke, M.A., Rongen, J.J., Overeem, S., Schelhaas, H.J., Zwarts, M.J. \& Stegeman, D.F. (2013) Cumulative effect of 5 daily sessions of theta burst stimulation on corticospinal excitability in amyotrophic lateral sclerosis. Muscle Nerve, 48, 733-738.

Murakami, T., Muller-Dahlhaus, F., Lu, M.K. \& Ziemann, U. (2012) Homeostatic metaplasticity of corticospinal excitatory and intracortical inhibitory neural circuits in human motor cortex. J Physiol, 590, 5765-5781.

Nettekoven, C., Volz, L.J., Kutscha, M., Pool, E.M., Rehme, A.K., Eickhoff, S.B., Fink, G.R. \& Grefkes, C. (2014) Dose-dependent effects of theta burst rTMS on cortical excitability and resting-state connectivity of the human motor system. J Neurosci, 34, 68496859.

Nettekoven, C., Volz, L.J., Leimbach, M., Pool, E.M., Rehme, A.K., Eickhoff, S.B., Fink, G.R. \& Grefkes, C. (2015) Inter-individual variability in cortical excitability and motor network connectivity following multiple blocks of rTMS. Neuroimage, 118, 209-218.

Ngomo, S., Leonard, G., Moffet, H. \& Mercier, C. (2012) Comparison of transcranial magnetic stimulation measures obtained at rest and under active conditions and their reliability. J Neurosci Methods, 205, 65-71.

Oldfield, R.C. (1971) The assessment and analysis of handedness: the Edinburgh inventory. Neuropsychologia, 9, 97-113.

Pitcher, J.B., Riley, A.M., Doeltgen, S.H., Kurylowicz, L., Rothwell, J.C., McAllister, S.M., Smith, A.E., Clow, A., Kennaway, D.J. \& Ridding, M.C. (2012) Physiological evidence

This article is protected by copyright. All rights reserved. 
consistent with reduced neuroplasticity in human adolescents born preterm. $J$ Neurosci, 32, 16410-16416.

Ridding, M.C. \& Ziemann, U. (2010) Determinants of the induction of cortical plasticity by non-invasive brain stimulation in healthy subjects. J Physiol, 588, 2291-2304.

Rossini, P.M., Burke, D., Chen, R., Cohen, L.G., Daskalakis, Z., Di lorio, R., Di Lazzaro, V., Ferreri, F., Fitzgerald, P.B., George, M.S., Hallett, M., Lefaucheur, J.P., Langguth, B., Matsumoto, H., Miniussi, C., Nitsche, M.A., Pascual-Leone, A., Paulus, W., Rossi, S., Rothwell, J.C., Siebner, H.R., Ugawa, Y., Walsh, V. \& Ziemann, U. (2015) Non-invasive electrical and magnetic stimulation of the brain, spinal cord, roots and peripheral nerves: Basic principles and procedures for routine clinical and research application. An updated report from an I.F.C.N. Committee. Clin Neurophysiol, 126, 1071-1107.

Rothwell, J.C. (1997) Techniques and mechanisms of action of transcranial stimulation of the human motor cortex. J Neurosci Methods, 74, 113-122.

Sale, M.V., Ridding, M.C. \& Nordstrom, M.A. (2008) Cortisol inhibits neuroplasticity induction in human motor cortex. J Neurosci, 28, 8285-8293.

Siebner, H.R. (2010) A primer on priming the human motor cortex. Clin Neurophysiol, 121, 461-463

Sinclair, C. \& Hammond, G.R. (2009) Excitatory and inhibitory processes in primary motor cortex during the foreperiod of a warned reaction time task are unrelated to response expectancy. Exp Brain Res, 194, 103-113.

Sommer, M., Wu, T., Tergau, F. \& Paulus, W. (2002) Intra- and interindividual variability of motor responses to repetitive transcranial magnetic stimulation. Clin Neurophysiol, 113, 265-269.

Suppa, A., Huang, Y.Z., Funke, K., Ridding, M.C., Cheeran, B., Di Lazzaro, V., Ziemann, U. \& Rothwell, J.C. (2016) Ten Years of Theta Burst Stimulation in Humans: Established Knowledge, Unknowns and Prospects. Brain Stimul, 9, 323-335.

Valero-Cabre, A., Pascual-Leone, A. \& Rushmore, R.J. (2008) Cumulative sessions of repetitive transcranial magnetic stimulation (rTMS) build up facilitation to subsequent TMS-mediated behavioural disruptions. Eur J Neurosci, 27, 765-774.

Vallence, A.M., Goldsworthy, M.R., Hodyl, N.A., Semmler, J.G., Pitcher, J.B. \& Ridding, M.C. (2015) Inter- and intra-subject variability of motor cortex plasticity following continuous theta-burst stimulation. Neuroscience, 304, 266-278.

Vernet, M., Bashir, S., Yoo, W.K., Oberman, L., Mizrahi, I., Ifert-Miller, F., Beck, C.J. \& Pascual-Leone, A. (2014) Reproducibility of the effects of theta burst stimulation on motor cortical plasticity in healthy participants. Clin Neurophysiol, 125, 320-326.

Ziemann, U \& Siebner, H.R. (2008) Modifying motor learning through gating and homeostatic metaplasticity. Brain Stimul, 1, 60-66.

This article is protected by copyright. All rights reserved. 


\section{Figure captions}

Figure 1. Experiment timeline. For each of the five days, RMT and AMT where measured first followed by SICI and ICF, which were only measured on days 1 and 5 . Then the baseline MEP amplitude was recorded by collecting 20 MEPs using a stimulator intensity of $120 \%$ of RMT. Then 600 pulses of either active or sham iTBS were delivered and followed by 7 blocks of 20 MEPs each immediately after iTBS, every 5 minutes up to 30 minutes.

Figure 2. Box-and-whisker plot of log transformed normalized average MEP amplitudes for all five days and both active (solid line) and sham (dashed line) iTBS groups. The whiskers of the plot represent the data points within 1.5 interquartile range of the lower and upper quartile. Values outside this range are plotted as individual black dots. There were no significant differences between the two groups.

Figure 3. SICl and ICF Box-and-whisker plot showing log transformed normalized average MEP amplitude, at 4 different ISIs. Groups are displayed side by side. The whiskers of the plot represent the data points within 1.5 interquartile range of the lower and upper quartile. Values outside this range are plotted as individual black dots. No significant differences between groups or days 1 and 5 were found.

Figure 4. Line plot showing log transformed normalized average MEP amplitude for each day. Each line corresponds to one participant.

This article is protected by copyright. All rights reserved. 
Table 1. Responders and non-responders in iTBS and sham groups, for $10 \%$ and $20 \%$ cutoff values.

\begin{tabular}{|c|c|c|c|c|c|c|c|c|c|c|c|c|c|}
\hline & & $10 \%$ & & & & & & $20 \%$ & & & & & \\
\hline & & Day 1 & Day 2 & Day 3 & Day 4 & Day 5 & Total & Day 1 & Day 2 & Day 3 & Day 4 & Day 5 & Total \\
\hline \multirow[t]{2}{*}{ iTBS } & Responders & 6 & 3 & 6 & 3 & 6 & 24 & 4 & 2 & 3 & 3 & 5 & 25 \\
\hline & Non-responders & $4[2]$ & $7[3]$ & $4[2]$ & $7[6]$ & $4[1]$ & 26 & $6[1]$ & $8[1]$ & $7[1]$ & $7[5]$ & $5[0]$ & 25 \\
\hline \multirow[t]{2}{*}{ Sham } & Responders & 7 & 4 & 7 & 6 & 9 & 33 & 5 & 4 & 4 & 5 & 8 & 26 \\
\hline & Non-responders & $3[1]$ & $6[4]$ & $3[1]$ & $4[2]$ & $1[0]$ & 17 & $5[1]$ & $6[2]$ & $6[1]$ & $5[2]$ & $2[0]$ & 24 \\
\hline
\end{tabular}

Values in brackets show the number of inhibitory non-responders.

Table 2. Average RMT and AMT for each group and day.

\begin{tabular}{lllllll} 
& & day 1 & day 2 & day 3 & day 4 & day 5 \\
\hline \multirow{2}{*}{ Active } & RMT & $46.1(9.2)$ & $44.5(9.6)$ & $45.5(9.7)$ & $45.3(9.3)$ & $46.2(8.4)$ \\
& AMT & $49.8(8.0)$ & $47.7(8.9)$ & $47.7(9.3)$ & $48.1(9.3)$ & $48.2(8.1)$ \\
\hline \multirow{2}{*}{ Sham } & RMT & $42.9(4.9)$ & $40.8(4.1)$ & $40.2(3.4)$ & $40.7(4.2)$ & $42.6(5.8)$ \\
& AMT & $50.0(11.4)$ & $49.1(8.8)$ & $47.6(8.2)$ & $46.3(6.6)$ & $51.0(11.6)$
\end{tabular}

Values correspond to the mean RMT and AMT stimulation intensities, expressed as a percentage of maximum output from the stimulator. Values in parenthesis correspond to the standard deviation. The overall higher intensities of AMT are due to the fact that it was measured using Magstim Rapid2, which produces biphasic pulse waveforms and delivers less power overall than the single pulse stimulator, hence the need for relatively higher intensities.

This article is protected by copyright. All rights reserved. 


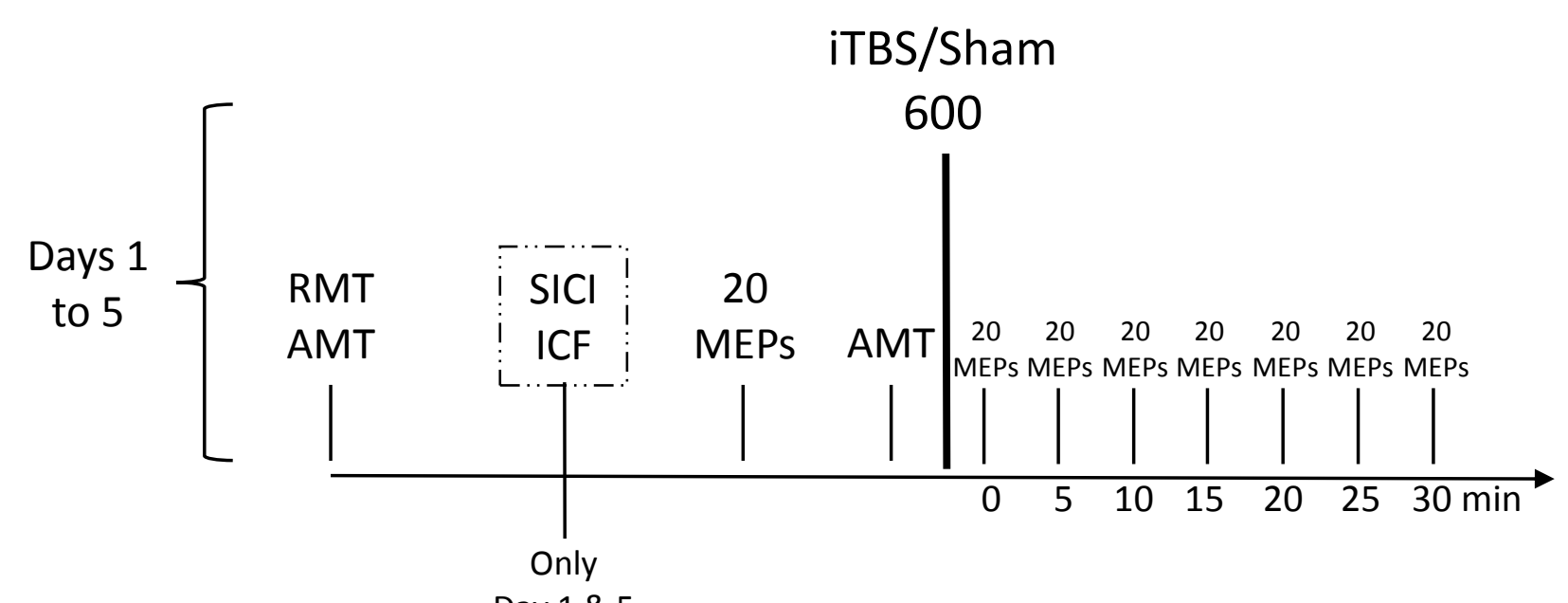

Day 1 \& 5

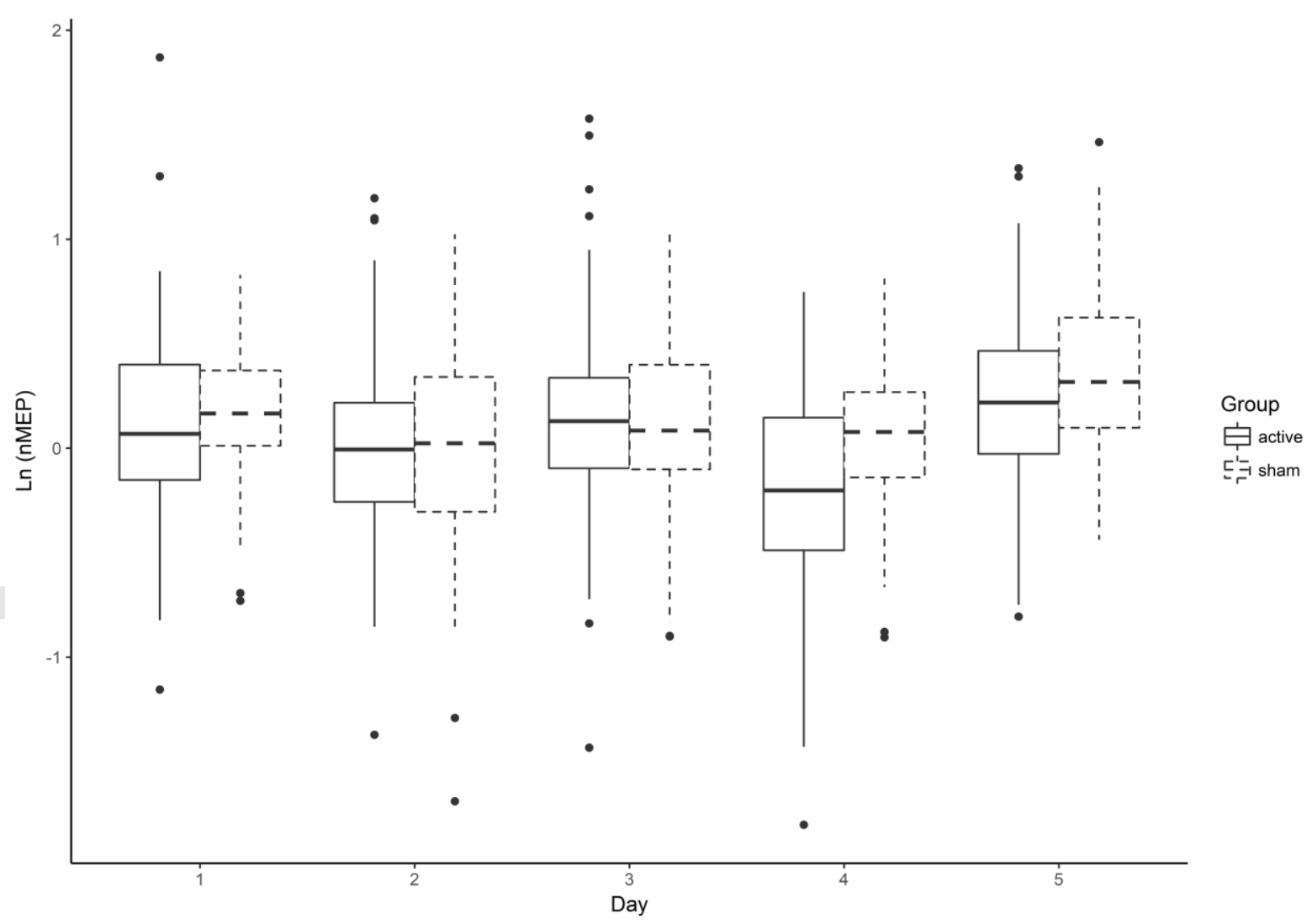

This article is protected by copyright. All rights reserved. 



This article is protected by copyright. All rights reserved. 